\title{
基于自适应分数阶微分的红外目标增强算法
}

\author{
代少升，李东阳，聂合文，姚 俐 \\ (重庆邮电大学 通信与信息工程学院, 重庆 400065)
}

\begin{abstract}
摘要: 针对红外图像存在灰度范围窄、图像细节不清晰、目标边缘模糊的问题, 提出了一种基于自适 应分数阶微分的红外目标增强方法。该方法首先利用图像的梯度、信息熵进行有效融合，并且自适应 调整分数阶微分以增强图像中的目标边缘; 然后采用图像像素灰度的标准差和均值进行融合去确定目 标的分割阈值, 以区分出图像中的背景和目标部分; 通过对图像中的目标区域进行线性增强, 以进一 步突显目标。经过实验验证: 本文提出的方法能够有效地区分红外图像中的目标和背景, 局部目标背 景比（Target-to-Background Ratio, TBR）平均提高了 0.5 , 视觉效果比较理想。
\end{abstract}

关键词: 红外图像; 目标增强; 自适应分数阶微分; 线性变换; 局部目标背景比

中图分类号：TP391 文献标志码：A 文章编号：1001-8891(2020)05-0257-07

\section{Linear Enhancement Algorithm of Infrared Target Based on Adaptive Fractional Differentiation}

\author{
DAI Shaosheng, LI Dongyang, NIE Hewen, YAO Li \\ (School of Communication and Information Engineering, \\ Chongqing University of Posts and Telecommunications, Chongqing 400065, China)
}

\begin{abstract}
To solve the problems associated with infrared images, such as narrow gray range, unclear image details and fuzzy target edge, an infrared target enhancement method based on adaptive fractional differentiation is proposed. In this method, first, the gradient and information entropy of image are used for effective fusion, and the fractional differentiation is adaptively adjusted to enhance the edge of the target in the image. Subsequently, the standard deviation and mean value of the image pixel gray are fused to determine the segmentation threshold of the target, to distinguish the background and target in the image. The target area of the image is linearly enhanced to better highlight the target. Experimental results show that the proposed method can effectively distinguish the target and background in the infrared image. The average local target-to-background ratio (TBR) increased by 0.5 , and the visual effect was ideal.
\end{abstract}

Key words: infrared image, target enhancement, adaptive fractional differentiation, linear transformation, local target-to-background ratio

\section{0 引言}

近些年来, 随着红外技术不断发展, 红外热成像 在军事和民用上得到广泛应用 ${ }^{[1]}$ 。由于红外图像多呈 现昏暗、模糊, 信噪比一般较低 ${ }^{[2]}$, 并且红外目标经 常被淹没在背景之中, 所以红外目标的增强作为红外 目标检测的前期工作尤为重要, 影响后续的红外目标 的检测以及跟踪 ${ }^{[3]}$ 。红外目标增强主要有点增强、空 域增强、频域增强 3 种类型。最早采用的点增强法主 要利用了图像的灰度变换和几何变换, 通过对比度增 强和对比度拉伸等方法达到增强图像目标的目的。比
如 Zhang 等人 ${ }^{[4]}$ 提出了一种对比度受限自适应直方图 均 衡 (Contrast Limited Adaptive Histogram Equalization, CLAHE) 算法, 对局部直方图的高度 进行限制, 以限制局部对比度的增强幅度; 虽然增强 了图像目标的对比度和限制了背景噪声的增加; 但是 也造成了目标增强程度的下降。另一方面, 红外目标 空域增强利用了图像的空间信息; 通过目标的位置、 形状、大小等特征区分目标和背景, 从而有效地增强 图像目标。在这方面, Luan 等人 ${ }^{[5]}$ 提出了一种多分辨 多尺度的红外图像增强算法, 利用目标的边缘轮廓特 征进行模糊融合聚类和多分辨小波分解; 虽然提高了 
图像信噪比并使得目标更为突出, 但是目标细节信息 的增强效果不好。除了点增强和空域增强, 人们也从 频域的角度研究了图像目标的增强。Qi 等人 ${ }^{[6]}$ 提出一 种基于四元傅里叶相位谱 (Phase Spectrum of Quaternion Fourier Transform, PQFT）的红外目标增强 方法, 利用四元傅里叶变换的相位谱和导数滤波器区 分目标和背景; 虽然增强了红外目标, 但是只适应于 小目标。

本文提出了一种基于自适应分数阶微分的红外 目标增强方法。为了解决红外目标整体对比度不高和 边缘不清晰的问题, 首先对红外图像进行自适应分数 阶微分滤波处理, 然后对红外图像进行局部目标线性 增强, 最后将两种目标增强效果融合。该算法不仅增 强了目标的边缘细节, 而且增加了目标的对比度和避 免了背景噪声的增加, 具有很好的红外目标增强视觉 效果。

\section{1 自适应分数阶微分}

\section{1 自适应参数融合}

为了更好地获得红外目标局部特征信息, 利用图 像的梯度和局部信息熵联合得到目标特征, 从而获得 整幅图像的动态参数。如图 1, 图像统一采用 $3 \times 3$ 的 模板进行矩形局部区域特征计算。目标区域梯度采用 图像的水平梯度、垂直梯度、主对角线梯度和副对角 线梯度联合获得, 所以目标包含 8 个方向的梯度特征。 图像的梯度 $G(i)$ 计算如下:

$$
G(i)=\frac{G_{\mathrm{h}}(i)}{4}+\frac{G_{\mathrm{v}}(i)}{4}+\frac{G_{\mathrm{p}}(i)}{4}+\frac{G_{\mathrm{c}}(i)}{4}
$$

式中: $G_{\mathrm{h}}(i)$ 是图像第 $i$ 点像素的水平梯度; $G_{\mathrm{v}}(i)$ 是图 像第 $i$ 点像素的垂直梯度; $G_{\mathrm{p}}(i)$ 是图像第 $i$ 点像素的 主对角线梯度; $G_{\mathrm{c}}(i)$ 是图像第 $i$ 点像素的副对角线梯 度。图像的局部熵 $E(i)$ 计算如下:

$$
\begin{gathered}
E(i)=-\sum_{j} P_{j} \log _{2} P_{j} \\
P_{j}=\operatorname{Hist}_{j} /\left(N^{*} N\right)
\end{gathered}
$$

式中: $1 \leqslant j \leqslant 256, j$ 是像素值; $N$ 是模板大小; $\mathrm{Hist}_{j}$ 是以第 $i$ 点像素为中心的模板图像经过局部直方图统 计所得的 $j$ 大小像素值的个数。图像的自适应特征参 数值大小对应于图像每个像素点的增强程度, 主要用 $0 \sim 1$ 范围的数值表示, 值越大, 增强程度越强。为了 使特征参数值映射到 $0 \sim 1$ 之内, 对以上两个特征参 数分别进行归一化处理:

$$
T(i)=\frac{T(i)-\operatorname{Min}(T(i))}{\operatorname{Max}(T(i))-\operatorname{Min}(T(i))}
$$

式中： $T(i)$ 是图像第 $i$ 个像素点的一个特征参数; $\operatorname{Max}(T(i))$ 是特征参数 $T(i)$ 的最大值; $\operatorname{Min}(T(i))$ 是特征 参数 $T(i)$ 的最小值。最后进行两个特征参数的融合。 由于两个特征所占权重大小差别不大, 所以采用求和 平均法融合, 计算公式如下:

$$
\operatorname{SIG}(i)=\frac{G(i)}{2}+\frac{E(i)}{2}
$$

式中: $G(i) 、 E(i)$ 分别是图像的梯度、局部商; $\operatorname{SIG}(i)$ 是图像每个像素点的局部融合特征值, 主要突显了图 像目标的边缘细节部分。

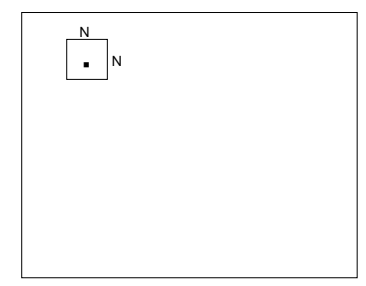

图 1 红外图像局部区域

Fig.1 Local area of infrared image

\section{2 分数阶微分增强}

分数阶微分是数学一个重要分支, 是由整数阶微 积分演化而来。为了准确地使用分数阶微分对图像处 理的计算, 采用 Grumwald-Letnikov ${ }^{[7]}$ 定义的分数阶微 分以増强图像细节。分数阶微分优于整数阶微分, 更 加有利于突出目标边界细节。分数阶微分使用由 G-L 推广得到的 $v$ 阶分数阶微分一元函数表达式:

$$
\begin{aligned}
\frac{\mathrm{d}^{v} f(t)}{\mathrm{d} t^{v}} \approx & f(t)+(-v) f(t-1)+\frac{(-v)(-v+1)}{2} f(t-2)+ \\
& \frac{-v(-v+1)(-v+2)}{6} f(t-3)+ \\
& \ldots+\frac{\Gamma(-v+1)}{(n-1) ! \Gamma(-v+n)} f(t-n-1)
\end{aligned}
$$

式中: $v$ 是分数阶微分的阶数; $f(t)$ 是一元函数; $n$ 个 系数 分别是 $1,-v, \frac{(-v)(-v+1)}{2}$, $\frac{-v(-v+1)(-v+2)}{6}, \cdots, \frac{\Gamma(-v+1)}{(n-1) ! \Gamma(-v+n)}$ 除了第一 个系数为 1 外, 其它系数都是阶数 $v$ 的函数。对于图 像每个像素, 为了充分利用局部周围像素信息进行增 
强处理, 采用以该像素点的 8 个方向进行分数阶微分 处理, 如图 2 所示。

整个图像采用模板进行卷积处理, 选择 $5 \times 5$ 的 模板和公式(6)的前 3 个系数重新构造模板, 如图 3。 由于采用 8 个方向进行增强, 所以将公式(6)的第一个 系数 1 乘以 8 得到 $a_{1}$ 。 $a_{1}, a_{2}, a_{3}$ 分别是 $8,-v$, $\frac{(-v)(-v+1)}{2}$ 。最后模板进行归一化操作, 整体除以 $(8-12 \times v+4 \times v \times v)$ 。

进行分数阶微分主要目的是增强图像高频部分, 保留图像的中低频部分。图像的高频部分具体指图像 的纹理和边缘部分, 其中也包括目标的边缘部分; 中 低频主要对应于图像背景平滑区域。当阶数 $v$ 增大, 图像的高频部分被增强; 然而当 $v$ 过大, 则过量増强 目标部分和过少保留中低频部分, 所以利用图像特征 参数自适应调节微分阶数 $v$ 的大小，并且 $v$ 的取值范 围在 $0 \sim 1$ 范围之间。图像每个像素的分数阶微分増 强阶数 $v(i)$ 赋值如下:

$$
v(i)=\operatorname{SIG}(i)
$$

式中: $\operatorname{SIG}(i)$ 是图像每个像素点的局部融合特征值。 最后对整个图像所有像素点进行自适应地模版卷积 滤波处理, 图像目标区域对应的像素点的 $\operatorname{SIG}(i)$ 较大, 对应的 $v(i)$ 也就较大, 增强效果较明显; 并且图像背 景区域对应的像素点的 $\operatorname{SIG}(i)$ 较小, $v(i)$ 也就较小, 较 好地保留背景信息。红外图像经过自适应分数阶微分 增强后的效果如图 4。在图 4(b)中, 目标边缘清晰和 很好地被增强。

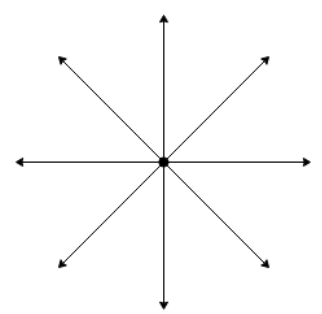

图 2 图像像素点的 8 个方向

Fig.2 Eight directions of image pixels

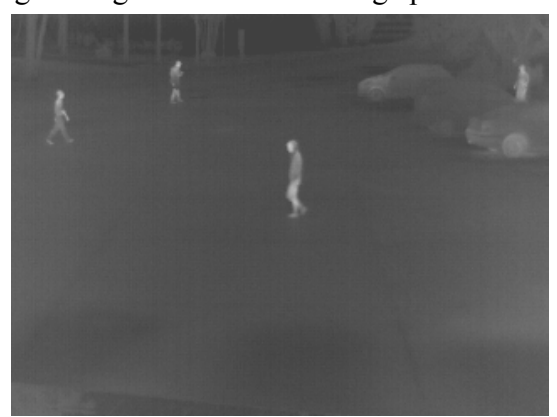

(a) 原图 (a) Original image

(b) 自适应分数阶微分

\begin{tabular}{|l|l|l|l|l|}
\hline$a_{3}$ & 0 & $a_{3}$ & 0 & $a_{3}$ \\
\hline 0 & $a_{2}$ & $a_{2}$ & $a_{2}$ & 0 \\
\hline$a_{3}$ & $a_{2}$ & $a_{1}$ & $a_{2}$ & $a_{3}$ \\
\hline 0 & $a_{2}$ & $a_{2}$ & $a_{2}$ & 0 \\
\hline$a_{3}$ & 0 & $a_{3}$ & 0 & $a_{3}$ \\
\hline
\end{tabular}

图 $35 \times 5$ 分数阶微分模板

Fig. $35 \times 5$ fractional order differential template

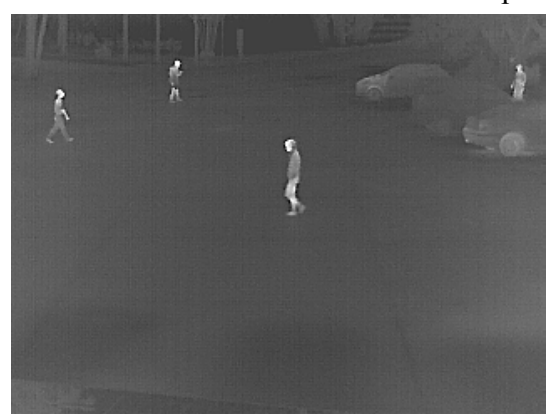

(b) Adaptive fractional differential

图 4 自适应分数阶微分增强

\footnotetext{
Fig.4 Adaptive fractional differential enhancement
}

\section{2 局部目标线性增强}

线性拉伸可以增强图像的对比度, 但是如果对整 幅图像拉伸会导致目标与背景同时增强, 目标增强效 果不好; 因此采用局部目标线性拉伸处理红外图像。 局部目标线性拉伸首先采用图 1 中的局部模板对红外 图像进行目标提取, 然后对所提取的目标区域再进行 线性变换处理。本文采用阀值判断法区别目标和背景 区域。根据图像的局部均值和局部标准差融合得到目 标特征值 CTR $(i)$, 图像的局部均值 $M(i)$ 和局部标准差

$$
\begin{gathered}
M(i)=\frac{1}{N^{*} N} \sum_{x} \sum_{y} f(x, y) \\
S(i)=\frac{1}{N} \sqrt{\sum_{x} \sum_{y}(f(x, y)-M(i))}
\end{gathered}
$$

式中: $x 、 y$ 的范围是 $x_{i}-\frac{N-1}{2} \leq x \leq x_{i}+\frac{N-1}{2}$, $y_{i}-\frac{N-1}{2} \leq y \leq y_{i}+\frac{N-1}{2} ; x 、 y$ 分别是像素点的横坐 标和纵坐标; $x_{i} 、 y_{i}$ 分别是图像第 $i$ 点像素的横坐标和 纵坐标; $N$ 是模板大小; $f(x, y)$ 是图像对应坐标像素点 的灰度。局部均值 $M(i)$ 和局部标准差 $S(i)$ 同样经过公 式(4)进行归一化运算, 然后融合得到目标特征值 CTR $(i)$ :

$$
\mathrm{CTR}(i)=k_{1} M(i)+k_{2} S(i)
$$


式中: $k_{1}$ 和 $k_{2}$ 是融合系数。由于目标的局部均值的权 重大于局部标准差, 所以目标大小分别选取为 0.7 和 0.3 。当 $\mathrm{CTR}(i)$ 高于一定阀值 $\mathrm{Th}$ 时, 如果图像第 $i$ 点 像素属于目标区域, 进行线性变换处理, 反之不进行 处理。图像的分割阀值 Th 是由目标特征值 CTR $(i)$ 采 用迭代法求得最佳数值, 适应于各种不同模糊程度的 图像。阀值 $\mathrm{Th}$ 主要计算方法是: 首先取出红外图像 目标特征值 CTR $(i)$ 的最大值和最小值, 进行求均值得 到初步阀值 $\mathrm{Tn}$; 然后将红外图像中目标特征值 CTR $(i)$ 大于阀值 $T n$ 的区域分割为一部分, 小于阀值 $T n$ 的区 域分割为另一部分; 分别求出两部分的图像区域像素 的目标特征值 $\operatorname{CTR}(i)$ 的均值, 再进行平均得到阀值 $\mathrm{Th}$; 如果 $\mathrm{Th}$ 和 $\mathrm{Tn}$ 的差值大于 0.005 , 将 $\mathrm{Th}$ 赋值给 $\mathrm{Tn}$ 继续重复以上步骤, 迭代优化直到取得目标和背景 的分割阀值 $\mathrm{Th}$ 的最佳值。最后对图像阀值分割后的 目标区域进行局部线性变换处理, 主要过程是首先对 阀值分割后的图像目标区域计算局部模板最大值 $m_{\max }(i)$ 和局部模板最小值 $m_{\min }(i)$, 计算如下:

$$
\begin{gathered}
m_{\max }(i)=\max \left(f_{\mathrm{m}}(i)\right) \\
m_{\min }(i)=\min \left(f_{\mathrm{m}}(i)\right)
\end{gathered}
$$

式中: $f_{\mathrm{m}}(i)$ 是以图像目标区域中第 $i$ 个像素点中心的模 板区域。然后进行像素线性区间变换, 目标区域的像 素值区间拉伸到局部模板最小值到 1 之间，计算如下：

$$
f(i)=\left\{\begin{array}{cc}
1 & m_{\text {max }}(i)=m_{\text {min }}(i) \\
T_{\mathrm{S}}(i) & \text { else }
\end{array}\right.
$$

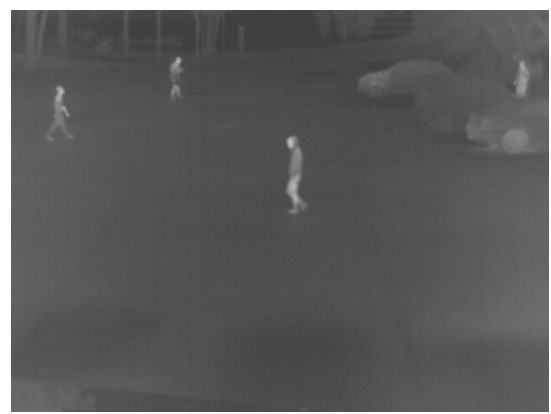

(a) 原图 (a) Original image

图 5 局部目标线性增强

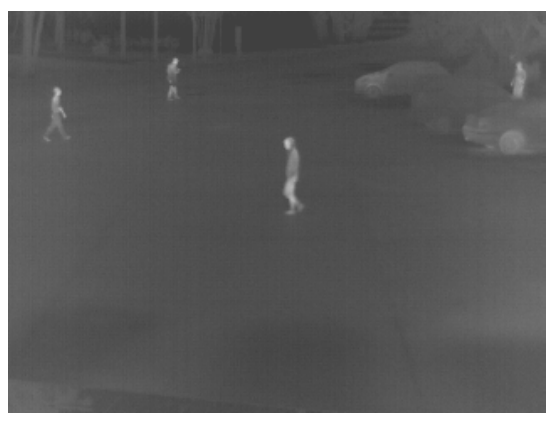

(a) 原图 (a) Original image

$$
\begin{aligned}
T_{\mathrm{S}}(i)= & m_{\text {min }}(i)+\frac{f(i)\left(1-m_{\min }(i)\right)}{m_{\text {max }}(i)-m_{\min }(i)}- \\
& \frac{m_{\text {min }}(i)\left(1-m_{\text {min }}(i)\right)}{m_{\text {max }}(i)-m_{\text {min }}(i)}
\end{aligned}
$$

式中: $T_{\mathrm{S}}(i)$ 是图像目标区域中第 $i$ 个像素点经过拉伸 后的像素值; $f(i)$ 是图像目标区域中第 $i$ 个像素点的像 素值。目标区域得到合适增强, 背景区域很好地被保 留，所以只有目标区域被拉伸和呈现高亮。局部目标 线性增强效果如图 5。

\section{3 目标增强算法融合}

红外图像在经过分数阶微分增强目标后, 虽然目 标边缘清晰, 但是目标内部对比度过低; 然而经过局 部目标线性增强后, 虽然目标内部得到拉伸, 但是目 标边缘不清晰。所以将两种方法处理后的结果进行叠 加融合, 为了更好地突出目标内部像素, 局部目标线 性增强后的结果占较大比重。对红外图像进行算法处 理后的对比图以及像素分布图如图 6。在图 6(b)中, 目标物体被明显增强, 亮度明显提升。分数阶微分增 强使边缘明显更加清晰，局部线性目标拉伸使目标明 亮较完整。通过对比图 6(c)和(d), 更能观察到目标整 体的像素值得到提高, 而目标周围背景几乎没有被增 强, 避免了大部分的背景干扰。

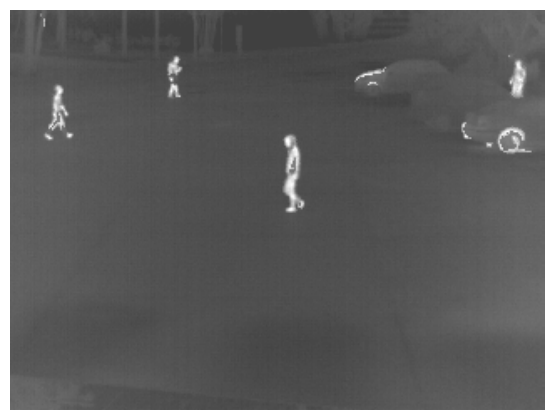

(b) 局部线性变换 (b) Local linear transformation

Fig.5 Local target linear enhancement

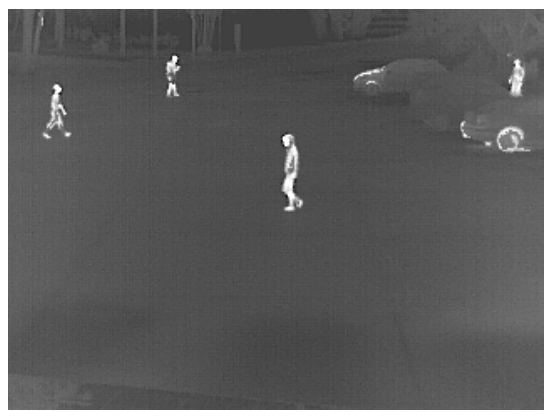

(b) 目标融合增强图像

(b) Target fusion enhancement image 

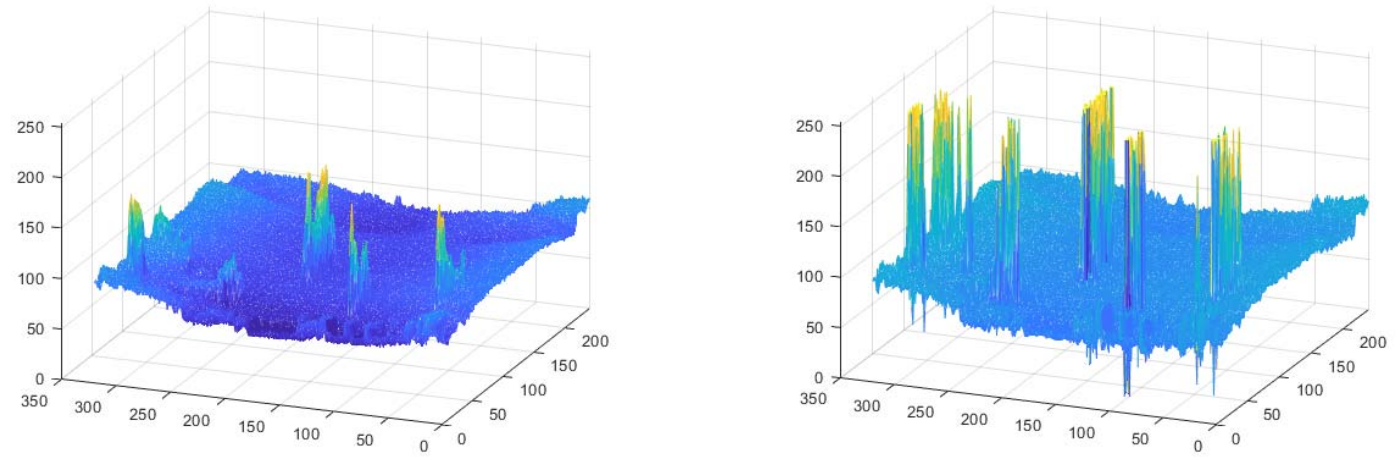

(c) 原像素分布 (c) Original pixel distribution (d) 目标融合增强后的像素分布 (d) Pixel distribution after target fusion enhancement 图 6 本文算法效果图 Fig.6 The effect image of this algorithm

\section{4 结果与分析}

为了分析本文算法的性能且突出在红外目标增 强的效果, 首先将自适应直方图均衡化 ${ }^{[8]}$ (Adaptive Histogram Equalization, AHE)、CLAHE、小波变换 ${ }^{[9]}$ (Wavelet Transform, WT) 和 PQFT 4 种不同的目标 增强算法和本文算法处理相同的红外图像进行对比; 然后通过局部 TBR 指标评价各种目标增强方法的优 劣。本文采用以森林和大地为背景的红外图像数据 集。图像的尺寸大小为 $240 \times 320$ 。图像包含两种目标, 分别是 4 个人体目标和少量汽车目标, 如图 7(a)。本 文所有算法仿真实验都使用同一台个人笔记本电脑, 电脑硬件性能为 Intel i3-3110M 2.3 GHz CPU 核心、 12 $\mathrm{G}$ 运行内存, 使用的系统为 windows7 64 位, 仿真软 件为 Matlab。

在图 7 中, 图 7(a) 是原始图像。图像昏暗, 目标 模糊且边界不清晰, 特别右侧人体目标和背景相近。 如图 7(b), 原图经过 AHE 算法处理后, 人和车两种 目标都得到增强, 但是背景同样也得到增强; 图像整 体亮度提高, 但是模糊不清晰。如图 7(c), CLAHE 算法相比于 AHE 算法, 背景区域噪声没有被增强; 虽然目标区域得到增强, 但是目标增强程度过小。如

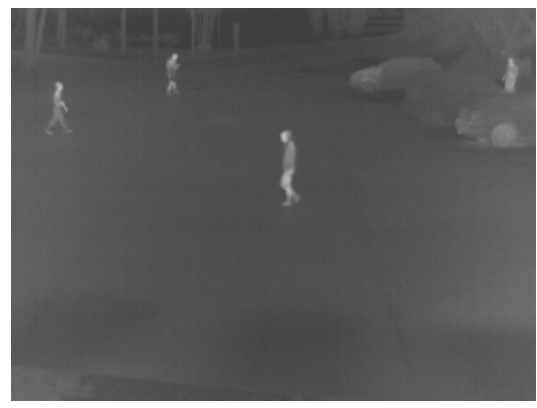

(a) 原图 (a) original graph
图 7(d), WT 算法处理后的图像目标明显被增强, 人 和车的一部分明亮突出, 但是图像背景过度增强导致 亮度过高且明显失真。图 7(e)是 PQFT 算法, 虽然图 像背景噪声得到很好地抑制并且背景保留完整和不 失真; 但是目标的边缘和整体增强效果不好。图 7(f) 是本文算法处理后的结果, 相比于以上 4 种算法, 图 像目标不仅明亮, 而且目标边缘清晰, 人体轮廓和肢 体部分明显区分, 车的部分细节十分清晰, 很好地保 留目标的特征; 图像背景也十分清晰, 噪声抑制效果 良好, 并且得到很好地保留, 没有出现失真。由以上 分析得出: 本文算法在一定程度上优于以上 4 种目标 增强算法。

为了全面地从数值上分析本文算法性能的优劣 性能, 使用局部 TBR 对比分析本文算法和其它 4 种 算法。局部 TBR 是目标区域和目标周围的局部背景 区域的像素均值之比, 从目标和目标周围背景的对比 程度反映红外图像目标增强的程度, 局部 TBR 计算 公式如下:

$$
\mathrm{TBR}=\frac{E_{\mathrm{T}}}{E_{\mathrm{B}}}
$$

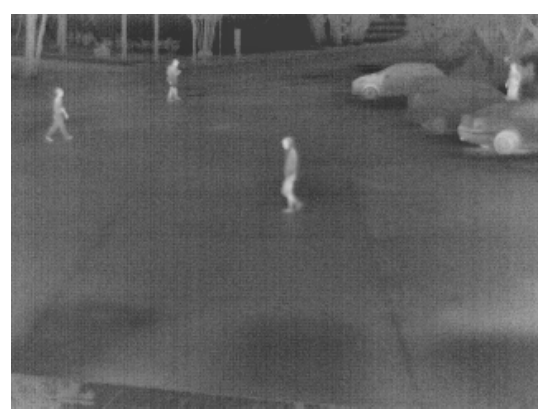

(b) AHE 


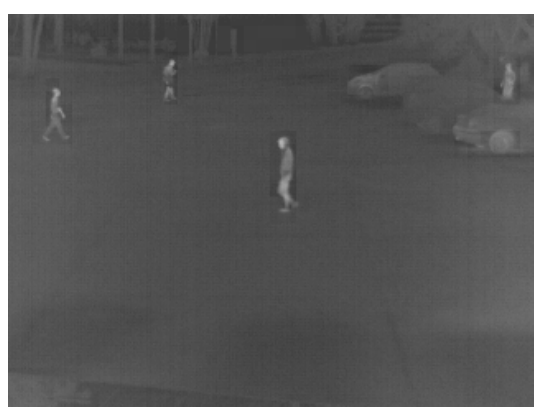

(c) CLAHE

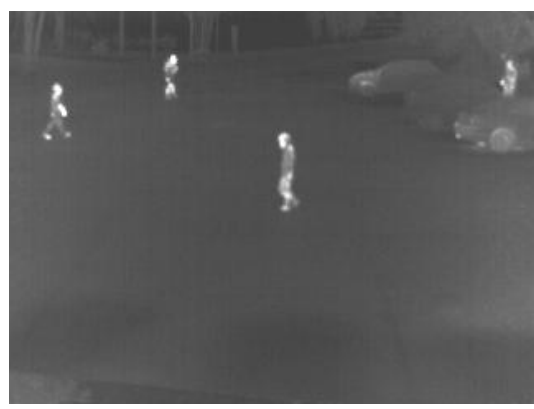

(e) PQFT

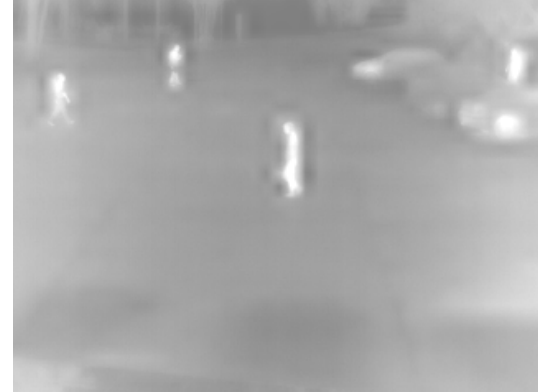

(d) WT

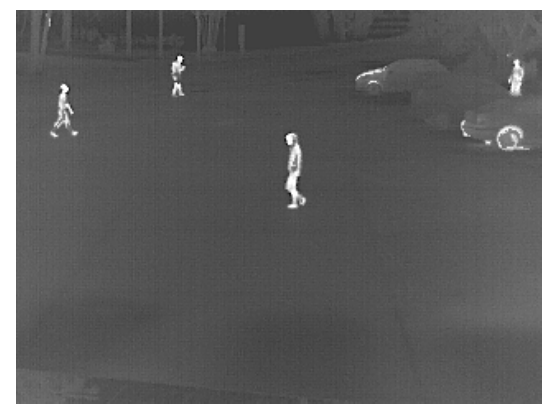

(f) 本文算法 (f) Algorithm in this paper

图 7 本文算法与 4 种图像目标增强算法对比图

Fig.7 Comparison between our algorithm and four image target enhancement algorithms

式中: $E_{\mathrm{T}}$ 是图像目标区域像素点灰度的均值; $E_{\mathrm{B}}$ 是 图像目标周围背景区域像素点灰度的均值。局部 TBR 的值越高, 说明该算法对红外图像目标增强的程度越 大。本文算法与 4 种目标增强算法分别在人和车的部 分两种目标的局部 TBR 值进行对比, 如表 1。在人体 目标的增强方面, 可以明显地得出本文算法局部 TBR 的值最大, 相比于其它 4 种算法, 平均提高 0.5 , 计 算公式如下:

$$
\begin{aligned}
& I_{\mathrm{avg}}=\frac{\mathrm{TBR}_{\mathrm{OUR}}-\mathrm{TBR}_{\mathrm{AHE}}}{4}+\frac{\mathrm{TBR}_{\mathrm{OUR}}-\mathrm{TBR}_{\mathrm{CLAHE}}}{4} \\
& +\frac{\mathrm{TBR}_{\mathrm{OUR}}-\mathrm{TBR}_{\mathrm{WT}}}{4}+\frac{\mathrm{TBR}_{\mathrm{OUR}}-\mathrm{TBR}_{\mathrm{PQFT}}}{4}
\end{aligned}
$$

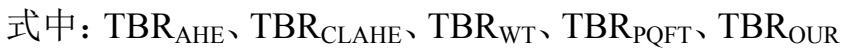
分别是 AHE、CLAHE、WT、PQFT 和本文算法的局 部 TBR 值, $I_{\text {avg }}$ 是本文算法相比其它 4 种算法局部 TBR 提高的平均值。所以本文算法对图像中的人体目 标增强程度最大。同样在车的部分增强方面, 除了 AHE 算法 TBR 的值优于 PQFT 算法外, 与图像人体 目标增强一样, 本文算法的 TBR 的值最大。最终说 明本文算法在红外图像目标增强程度效果最佳。综合 以上所有实验结果得出, 本文算法有效地增强了红外 图像目标, 优于其它 4 种目标增强算法。
表 1 不同算法对于不同目标的局部 TBR

Table 1 Local TBRs of different algorithms for different targets

\begin{tabular}{llllll}
\hline & AHE & CLAHE & WT & PQFT & OUR \\
\hline Person & 1.56 & 1.28 & 1.18 & 1.66 & 2.00 \\
Car & 1.24 & 1.05 & 0.98 & 1.17 & 1.63 \\
\hline
\end{tabular}

\section{5 结论}

本文提出了一种新的红外目标增强方法, 主要解 决红外图像的目标边缘细节不清晰和对比度低的问 题。首先利用了图像的梯度和局部信息摘联合得到新 的目标边缘特征, 作为阶数调整分数阶微分, 实现了 分数阶微分自适应增强图像目标; 目标的边缘细节得 到很好地增强, 并且目标本身和图像背景更加清晰。 然后实现了一种局部线性目标增强, 由图像的局部均 值和局部标准差融合得到的目标整体特征再进行自 适应迭代化处理; 分割出图像中的目标区域后，采用 线性变换增强目标内部, 得到的图像目标整体增强效 果较好。经过实验结果表明, 本文算法能够有效地区 分红外图像中的目标和背景, 适用于红外目标的增 强。 


\section{参考文献:}

[1] 吴旭景, 杜斌. 红外热成像无损检测技术现状及发展的相关研究 [J]. 化工管理, 2018(29): 183.

WU X J, DU B. Research on the status and development of Infrared Thermal Imaging Nondestructive Testing Technology[J]. Chemical Management, 2018(29): 183.

[2] 王好贤, 董衡, 周志权. 红外单帧图像弱小目标检测技术综述 [J]. 激 光与光电子学进展, 2019, 56(8): 9-22.

WANG H X, DONG H, ZHOU Z Q. Review on dim small target detection technologies in infrared single frame image[J]. Laser \& Optoelectronics Progress, 2019, 56(8): 9-22.

[3] 易诗, 张洋溢, 聂炎, 等. 红外图像中快速运动目标的检测与跟踪方 法[J]. 红外技术, 2019, 41(3): 268-272.

YI Shi, ZHANG Yangyi, NIE Yan, et al. Fast moving target detection and tracking method in infrared image[J]. Infrared Technology, 2019, 41(3): 268-272.

[4] 张丽. 对比度受限自适应直方图均衡方法 [J]. 电脑知识与技术, 2010, 6(9): 2238-2241.

ZHANG L. Contrast limited adaptive histogram equalization[J]. Computer Knowledge and Technology, 2010, 6(9): 2238-2241.

[5] 亱孟杰. 一种多分辨多尺度的红外图像增强算法 [J]. 激光杂志, 2019, 40(8): 81-84.
LUAN M J. A multiresolution and multiscale infrared image enhancement algorithm[J]. Laser Journal, 2019, 40(8): 81-84.

[6] QI S X, MA J, LI H, et al. Infrared small target enhancement via phase spectrum of Quaternion Fourier Transform[J]. Infrared Physics and Technology, 2014, 62: 50-58.

[7] 牛为华, 孟建良, 崔克涁, 等. 利用 Grümwald-Letnikov 分数阶方向导 数的图像增强方法 $[\mathrm{J}]$. 计算机辅助设计与图形学学报, 2016, 28(1): $129-137$.

NIU W H, MENG J L, CUI K B, et al. Image enhancement method using Grümwald-Letnikov fractional directional derivative[J]. Journal of Computer-Aided Design \& Computer Graphics, 2016, 28(1): 129-137.

[8] 刘德全, 崔涛, 杨雅宁. 局部对比度自适应直方图均衡化图像增强的 算法研究 $[J]$. 信息与电脑(理论版), 2016(7): 79-80.

LIU D Q, CUI T, YANG Y N. Algorithm Research on local contrast adaptive histogram equalization image enhancement $[\mathrm{J}]$. China Computer \& Communication (theoretical edition), 2016(7): 79-80.

[9] 王瑞. 小波变换在红外图像处理中的应用研究[D]. 淮南: 安徽理工大 学, 2016.

WANG R. The application of wavelet transform in infrared image processing[D]. Huainan: Anhui University of technology, 2016. 